\title{
PROCESSOS AVANÇADOS DE REMOÇÃO DE CIANOBACTÉRIA E CIANOTOXINA NO TRATAMENTO DE ÁGUA DE ABASTECIMENTO
}

\author{
F. P. CAMACHO ${ }^{1}$, L. O. R. MORETI ${ }^{1}$, F. S. ARAKAWA ${ }^{1}$, Q. L. SHIMABUKU ${ }^{1}$, M. F. \\ SILVA $^{1}$, T. R.T. SANTOS ${ }^{1}$, S. L. BAZANA ${ }^{1}$, P. F. COLDEBELLA ${ }^{1}$, K. C. VALVERDE ${ }^{1}$, \\ R. BERGAMASCO ${ }^{1}$
}

\begin{abstract}
${ }^{1}$ Universidade Estadual de Maringá, Departamento de Engenharia Química e-mail para contato: franciele_camacho@hotmail.com
\end{abstract}

\section{RESUMO}

O presente trabalho teve como objetivo avaliar a eficiência dos processos de coagulação/floculação/flotação por ar dissolvido (C/F/FAD) utilizando a sementes de Moringa oleifera, como coagulante natural, seguido de nanofiltração para remoção das células de cianobactéria e cianotoxina. Para os ensaios, foi preparada uma "água sintética", utilizando-se água deionizada artificialmente contaminada com células da espécie Microcystis protocystis para obter densidade celular na ordem de $10^{6}$ à $10^{7}$ cel $\cdot \mathrm{mL}^{-1}$. Observou-se que o uso da semente de Moringa oleifera promoveu a formação de flocos com menor densidade e diâmetro, favorecendo sua remoção na etapa de $\mathrm{C} / \mathrm{F} / \mathrm{FAD}$, além de possibilitar o aumento na eficiência da etapa de filtração. Em relação à NF, verificou-se uma remoção total das células de $M$. protocystis e da microcistina avaliada, indicando que a Moringa oleifera, não provocou a lise celular durante as etapas de tratamento.

\section{INTRODUÇÃO}

Os processos convencionais de tratamento de água, envolvendo normalmente as etapas de coagulação, floculação, sedimentação e filtração, se mostram eficientes na remoção de células de cianobactérias, no entanto, estes processos são considerados pouco eficientes em relação à remoção de cianotoxinas, apresentando valores baixos ou inexistentes de remoção. Assim, a água potável pode ser uma das principais fontes de exposição do homem às cianotoxinas. Neste contexto, um tratamento avançado precisa ser considerado para remoção desse composto dissolvido na água.

Os processos de separação por membranas vêm, cada vez mais, tornando-se importantes como alternativas aos processos de filtração convencionais. Dentre os diferentes processos de separação por membranas, a nanofiltração (NF), tem sido estudada como um processo promissor na remoção de cianotoxinas, por apresentar baixa porosidade, entre 0,001 e $0,01 \mu \mathrm{m}$, sendo capazes de reter compostos moleculares de 200 a $1.000 \mathrm{Da}$ (Schneider e Tsutiya, 2001), valores em que se inclui grande parte das cianotoxinas, como a microcistina-LR e a saxitoxina dc-STX.

Neste sentido, o presente trabalho tem como objetivo avaliar a capacidade de remoção de células de Microcystis protocystis por meio do emprego da flotação por ar dissolvido, utilizando a Moringa oleifera como coagulante natural, seguido de nanofiltração para remoção de células de cianobactéria e cianotoxinas, visando obter água dentro dos limites da legislação. 


\section{MATERIAIS E MÉTODOS}

\section{1 - Preparação da solução de Moringa oleifera $\left(\mathrm{E}_{\mathrm{s}}\right) \operatorname{com} \mathrm{NaCl}$ e $\mathrm{KCl}(1 \mathrm{M})$}

A solução de Moringa oleifera extraída foi preparada utilizando $1 \mathrm{~g}$ de polpa de semente da Moringa oleifera, descascadas e trituradas no liquidificador em $100 \mathrm{~mL}$ de solução salina de $\mathrm{NaCl}$ e $\mathrm{KCl}$ a $1 \mathrm{M}$. Optou-se por utilizar a concentração $1 \mathrm{M}$ do sal de $\mathrm{NaCl}$ e $\mathrm{KCl}$ devido a estudos anteriores aplicando ao tratamento de água (Madrona et al., 2010; M.C. Bongiovani et al., 2014).

Após a trituração no liquidificador, a solução foi agitada por 30 min e filtrada a vácuo. A extração sempre foi realizada no momento do ensaio de coagulação/floculação, pois estudos demonstraram que o armazenamento dessa solução por alguns dias pode ocasionar perda de eficiência (Cardoso, 2008).

A partir dessa solução foram usados 12 níveis dosagens: $25 \mathrm{mg} / \mathrm{L}, 50 \mathrm{mg} / \mathrm{L}, 75$ $\mathrm{mg} / \mathrm{L}, 100 \mathrm{mg} / \mathrm{L}, 125 \mathrm{mg} / \mathrm{L}, 150 \mathrm{mg} / \mathrm{L}, 175 \mathrm{mg} / \mathrm{L}, 200 \mathrm{mg} / \mathrm{L}, 225 \mathrm{mg} / \mathrm{L}, 250 \mathrm{mg} / \mathrm{L}, 275$ $\mathrm{mg} / \mathrm{L}$ e $300 \mathrm{mg} / \mathrm{L}$ (Madrona et al, 2010).

\section{2 - Ensaios de coagulação/floculação/flotação por ar dissolvido (C/F/FAD)}

A flotação por ar dissolvido foi avaliada nesse trabalho como método de separação, por se mostrar efetiva no tratamento de águas com elevada concentração de cianobactérias (Janssens e Buekens, 1993). O equipamento utilizado foi o floteste (Nova Ética - modelo 218/3) que é composto por uma câmara de saturação, conjunto motoragitador do Jar Test, três jarros confeccionados em acrílico transparente com dimensões de 115 x $11 \mathrm{~mm}$, cada um com capacidade para $2 \mathrm{~L}$.

Os parâmetros operacionais utilizados foram tempo de saturação $\left(\mathrm{T}_{\text {sat }}=8 \mathrm{~min}\right)$, pressão de saturação $\left(\mathrm{P}_{\text {sat }}=5\right.$ bar), taxa de recirculação $\mathrm{R}=8 \%$. (Ribau Teixeira e Rosa, 2006a).

\section{3 - Processos de Nanofiltração}

Nesta etapa, foram avaliadas as membranas NF-90 e NF-270 (Dow Chemical), quanto a sua eficiência na remoção de cianotoxinas. As membranas apresentam as mesmas configurações básicas, segundo López-Muñoz et al. (2009) e Nghiem e Hawkes (2009).

Para ter uma uniformidade nos ensaios fixou-se o volume inicial da alimentação de 5 litros, tempo de ensaio de 60 min e pressão de operação de 5 bar. Pressões maiores não foram avaliadas em função da capacidade do módulo de membrana. No equipamento a membrana foi disposta entre duas telas metálicas. A tela superior encontra-se na parte interna do corpo do dispositivo e serve como suporte para a barra metálica, que tem como função evitar a deposição de materiais suspensos sobre a membrana, o que pode reduzir o fluxo do permeado e o tempo de vida útil da membrana. A área de filtração da membrana é calculada em função do número de orifícios existentes na tela metálica inferior e da área individual de cada orifício.

Para todos os ensaios, realizou-se inicialmente a compactação da membrana com água deionizada e posteriormente a filtração da água "sintética" com cianotoxinas (microcistina), com o intuito de verificar a possível ocorrência de fouling na membrana.

Após a realização dos testes, a membrana foi caracterizada por MEV. Esta análise tem como objetivo a caracterização dos incrustantes na superfície da membrana. Para este teste, foi utilizado o Miscroscópico Eletrônico de Varredura FEI 
Company Quanta 200 com Micro Análise Oxiford Instruments - Penta FETx3.

\section{RESULTADO E DISCUSSÃO}

Considerando que as cianobactérias são capazes de liberar suas toxinas durante as etapas do tratamento de água e que a capacidade do tratamento convencional de água em remover essas cianotoxinas parece ser insuficiente, vários estudos vêm sendo realizados com o desenvolvimento de métodos mais avançados para remover as cianotoxinas dissolvidas na água.

Para verificar a eficiência na remoção de microcistina pelo processo proposto, as cianotoxinas foram avaliadas nos processos de $\mathrm{C} / \mathrm{F} / \mathrm{FAD}$ e combinado $\mathrm{C} / \mathrm{F} / \mathrm{FAD}+$ nanofiltração, utilizando as membranas NF-90 e NF-270. Os processos de C/F/FAD foram realizados com as dosagens ótima de Moringa $\mathrm{E}_{\mathrm{s}}$ com $\mathrm{NaCl}$ e $\mathrm{KCl}(1 \mathrm{M})$. Para os ensaios, a concentração inicial de microcistina foi de $10 \mu \mathrm{g} \cdot 1^{-1}$ para águas com densidade celular $10^{6}$ à $10^{7} \mathrm{cel} \cdot \mathrm{mL}^{-1}$.

Para o processo combinado com nanofiltração, além da análise de microcistina também foram realizados avaliação do fluxo do permeado e do fouling da membrana.

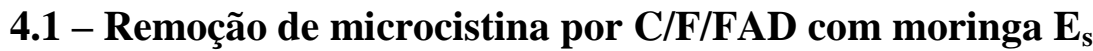

Quando a C/F/FAD foi aplicada usando a Moringa $\mathrm{E}_{\mathrm{s}}$ com $\mathrm{NaCl}$ e $\mathrm{KCl}$ a eficiência de remoção de microcistina foi $>52,1 \%\left(10^{6} \mathrm{cel} \cdot \mathrm{mL}^{-1}\right) \mathrm{e}>36,7 \%\left(10^{7} \mathrm{cel} \cdot \mathrm{mL}^{-}\right.$ 1) para solução com $\mathrm{NaCl}$ e $>41,3 \%\left(10^{7} \mathrm{cel} \cdot \mathrm{mL}^{-1}\right)$ e $>28,7 \%\left(10^{7} \mathrm{cel} \cdot \mathrm{mL}^{-1}\right)$ para a solução $\mathrm{KCl}$, como observada na Tabela 1 .

Tabela 1 - Eficiência da remoção de microcistina para o coagulante Moringa $\mathrm{E}_{\mathrm{s}}$ com $\mathrm{NaCl}$ e $\mathrm{KCl}(1 \mathrm{M})$ a $25 \mathrm{mg} / \mathrm{L}$ para densidade celular $10^{6}$ à $10^{7} \mathrm{cel} \cdot \mathrm{mL}^{-1}$.

\begin{tabular}{ccc}
\hline \multirow{2}{*}{$\begin{array}{c}\text { Densidade celular } \\
(\text { cél } / \mathbf{m L})\end{array}$} & \multicolumn{2}{c}{ Remoção de Microcistina $(\%)$} \\
\cline { 2 - 3 } & $\mathbf{E}_{\mathrm{s}} \mathbf{c o m} \mathbf{~ N a C l}$ & $\mathbf{E}_{\mathrm{s}}$ com KCl \\
\hline $\mathbf{1 0}^{\mathbf{6}}$ & 52,1 & 41,3 \\
$\mathbf{1 0}^{7}$ & 36,7 & 28,7 \\
\hline
\end{tabular}

$\mathrm{O}$ estudo mostrou que o processo de C/F/FAD usando coagulante natural Moringa tem sido eficiente na remoção de células de $M$. protocystis, mas não eficiente na remoção de microcistina na água após o pré-tratamento. Porém, quando comparados com os coagulantes químicos, pode-se observar que a utilização da Moringa não promoveu a lise celular após a adição do coagulante.

O mesmo comportamento de remoção da microcistina no presente estudo foi observado em outros trabalhos utilizando coagulantes químicos. Assis (2006), em estudo visando à remoção de $M$. aeroginosa e microcistina por FAD, obteve valores de remoção de toxinas extracelular relativamente baixa, entre 30 e $50 \%$, utilizando o sulfato de alumínio como coagulante e remoção praticamente desprezível quando da utilização de cloreto férrico. Ribau Teixeira e Rosa (2006a) obtiveram baixa eficiência de remoções de microcistina, aproximadamente $4,7 \%$ com a FAD, tendo sido observado liberação de toxinas para o meio em função do processo. A diferença nesses valores deve considerar as características da água a ser tratada, coagulante utilizado e demais fatores que possam interferir no tratamento. Não foram identificados estudos na 
literatura que avaliasse a remoção de microcistina utilizando a Moringa oleifera como coagulante pela flotação por ar dissolvido.

Considerando a reduzida eficiência da FAD para remoção de cianotoxinas e a possibilidade de liberação desta para o meio, quando da ocorrência de florações de elevada densidade, estudos que possibilitem a adoção de outros processos de tratamento após a FAD tornam-se necessários e relevantes do ponto de vista da qualidade da água tratada e destinada ao abastecimento.

Assim, como não foi possível obter água bruta com microcistina dentro dos limites exigidos pela legislação, utilizou-se a combinação com o processo de nanofiltração.

A literatura menciona os processos de separação com membranas como uma tecnologia promissora na obtenção de elevadas eficiências de remoção, tanto de cianobactéria como de cianotoxinas (Chorus, Bartram, 1999; Hitzfeld, Hoger, Dietric, 2000, Coral, 2009).

\section{2 - Associação da C/F/FAD e da NF para remoção de microcistina}

A Tabela 2 apresenta as eficiências de remoção da microcistina através do processo combinado $\mathrm{C} / \mathrm{F} / \mathrm{FAD}+\mathrm{NF}$ com solução salina de $\mathrm{NaCl}$ e $\mathrm{KCl}$ (1M), seguido por nanofiltração com as membranas NF-90 e NF270.

Tabela 2 - Eficiência de remoção da microcistina para as membranas NF-90 e NF-270, a partir da associação dos processos de $\mathrm{C} / \mathrm{F} / \mathrm{FAD}+\mathrm{NF}$

\begin{tabular}{|c|c|c|c|c|}
\hline \multirow{2}{*}{ Membranas } & \multirow{2}{*}{$\begin{array}{c}\text { Tempo } \\
\text { (min) }\end{array}$} & \multirow{2}{*}{$\begin{array}{c}\text { Microcistina } \\
\left(\mu \text { g. }^{-1}\right)\end{array}$} & \multicolumn{2}{|c|}{ Remoção com o pré-tratamento (\%) } \\
\hline & & & FAD com $\mathrm{NaCl}^{\mathrm{b}}$ & FAD com $\mathrm{KCl}^{\mathrm{b}}$ \\
\hline \multirow{4}{*}{ NF- 90} & Inicial $^{\mathrm{a}}$ & 10,0 & - & - \\
\hline & $\mathbf{t}=\mathbf{0}$ & 0,00 & 100,0 & 100,0 \\
\hline & $\mathbf{t}=\mathbf{3 0}$ & 0,00 & 100,0 & 100,0 \\
\hline & $t=60$ & 0.00 & 100,0 & 100,0 \\
\hline \multirow{4}{*}{$\mathrm{NF}-\mathbf{2 7 0}$} & Inicial & 10,0 & - & - \\
\hline & $\mathbf{t}=\mathbf{0}$ & 0,00 & 100,0 & 100,0 \\
\hline & $\mathbf{t}=\mathbf{3 0}$ & 0,00 & 100,0 & 100,0 \\
\hline & $t=60$ & 0,00 & 100,0 & 100,0 \\
\hline
\end{tabular}

Inicial $^{\text {a }}$ : concentração antes da C/F/FAD.

$\mathrm{FAD}+\mathrm{E}_{\mathrm{s}}$ com $\mathrm{NaCl}^{\mathrm{b}}$ : solução salina de Moringa (NaCl-1M)/ FAD

$\mathrm{FAD}+\mathrm{E}_{\mathrm{s}}$ com $\mathrm{KCl}^{\mathrm{b}}$ : solução salina de Moringa $(\mathrm{KCl}-1 \mathrm{M}) / \mathrm{FAD}$

Aplicando os processos combinados obteve-se uma remoção total da microcistina, atendendo os padrões da Portaria MS. 2.914/2011, que estabelece um limite máximo de $1 \mu \mathrm{g} / \mathrm{L}$ para água de consumo humano. Segundo Baker (2004), a nanofiltração (NF) consegue reter compostos de 200 a $1.000 \mathrm{Da}$, valores em que se inclui grande parte das cianotoxinas, como a microcistina que apresentam de $980 \mathrm{Da}$.

O mesmo comportamento foi observado por Ribau Teixeira e Rosa (2006b) conduziram um estudo visando avaliar a associação do sistema de flotação por ar dissolvido e nanofiltração para a remoção de microcistinas. Para tanto, os autores procederam às etapas de coagulação, empregando como coagulante o polihidroxiclorosulfato de alumínio, floculação, flotação (por meio de ar dissolvido ou pela relação $\mathrm{CO}_{2} /$ ar) e a nanofiltração. Ao final do experimento, verificou-se remoção total das cianobactérias, para ambos os processos de flotação, e remoção entre 99,4 e 99,7\% de Microcistina-LR, utilizando-se a nanofiltração como meio de separação.

A Tabela 3, apresenta a porcentagem de remoção de células de $M$. protocystis, turbidez e cor, respectivamente, após o processo combinado de $\mathrm{C} / \mathrm{F} / \mathrm{FAD}+\mathrm{NF}$ utilizando a solução salina com $\mathrm{NaCl}$ e $\mathrm{KCl}(1 \mathrm{M})$. 
Tabela 3 - Eficiência de remoção de células de $M$. protocystis, turbidez e cor pelo processo combinado $\mathrm{C} / \mathrm{F} / \mathrm{FAD}+\mathrm{NF}$

\begin{tabular}{|c|c|c|c|c|c|}
\hline Parâmetros & Inicial $^{*}$ & $\begin{array}{c}\mathbf{E}(\%)^{* *} \\
\text { FAD }+\mathrm{NF}-90^{\mathrm{a}}\end{array}$ & $\begin{array}{c}\mathrm{E}(\%) \\
\text { FAD+NF-270 }\end{array}$ & $\begin{array}{c}\text { E }(\%) \\
\text { FAD +NF-90 }\end{array}$ & $\begin{array}{c}\mathrm{E}(\%) \\
\text { FAD+NF-270 }\end{array}$ \\
\hline $\begin{array}{c}\text { Células de } \\
M . \text { protocystis }\end{array}$ & $1 \times 10^{6}\left(\mathrm{cel} \cdot \mathrm{mL}^{-1}\right)$ & 99,00 & 98,09 & 98,12 & 98,22 \\
\hline Turbidez (NTU) & $49-50 \pm 0,30$ & 98,50 & 97,10 & 99,70 & 99,31 \\
\hline Cor $(\mathrm{uH})$ & $754-755 \pm 0,35$ & 98,60 & 99,99 & 98,10 & 98,20 \\
\hline $\begin{array}{l}\text { Células de } \\
\text { M. protocystis }\end{array}$ & $1 \times 10^{7}\left(\mathrm{cel} \cdot \mathrm{mL}^{-1}\right)$ & 97,10 & 98,60 & 99,78 & 99,70 \\
\hline Turbidez (NTU) & $449-450 \pm 0,33$ & 98,00 & 98,08 & 98,45 & 98,77 \\
\hline Cor $(\mathrm{uH})$ & $1251-1250 \pm 0,39$ & 99,20 & 99,05 & 99,30 & 99,11 \\
\hline
\end{tabular}

Inicial*: "água sintética" sem tratamento; $\mathrm{E}(\%)^{* *}$ : porcentagem de remoção

$\mathrm{C} / \mathrm{F} / \mathrm{FAD}+\mathrm{NF} 90^{\mathrm{a}}$ e C/F/FAD + NF270': solução salina de Moringa (NaCl-1M)

$\mathrm{C} / \mathrm{F} / \mathrm{FAD}+\mathrm{NF}^{\mathrm{b}}$ e C/F/FAD + NF270' : solução salina de Moringa (KCl-1M)

Pode-se observar através da Tabela 3, que os parâmetros analisados se mostraram, após o tratamento combinado de C/F/FAD+NF, dentro dos padrões previstos pela legislação, independente da densidade celular da água bruta.

Avaliação do fluxo do permeado utilizando as membranas N-90 e NF-270 nas concentrações otimizadas de moringa em solução salina com $\mathrm{NaCl}(25 \mathrm{mg} / \mathrm{L})$ e $\mathrm{KCl}(25$ mg/L) estão apresentado na Figura 1 e 2.

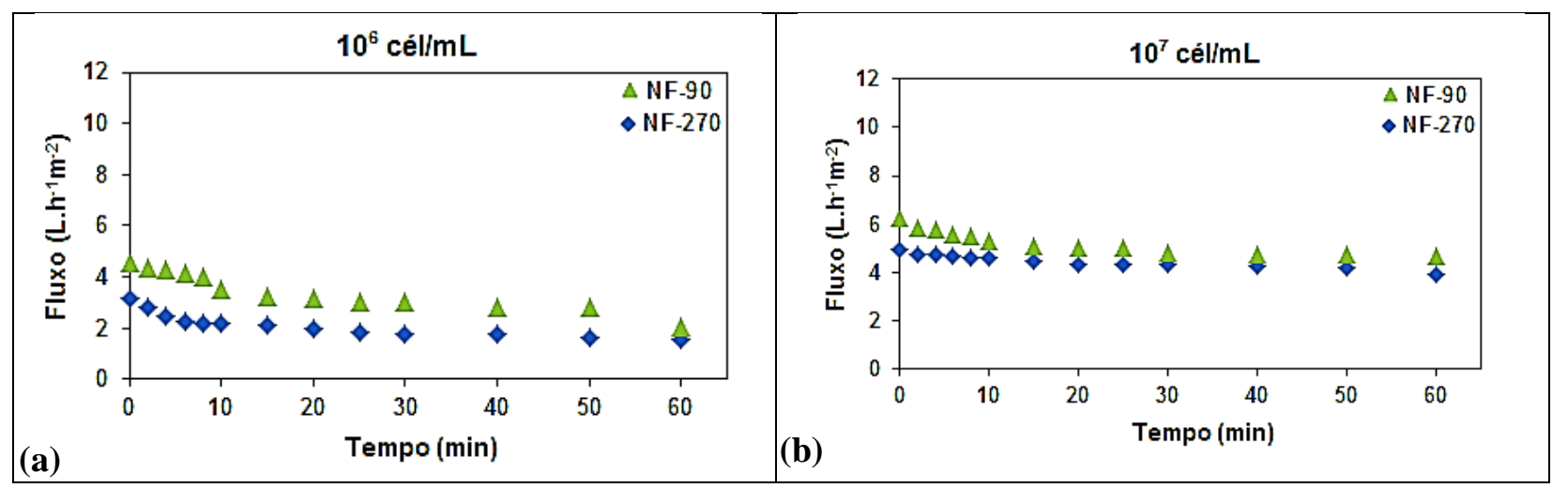

Figura 1 - Fluxo do permeado na pressão de 5 bar utilizando a Moringa $\mathrm{E}_{\mathrm{s}}$ com $\mathrm{NaCl}(1 \mathrm{M})$ para as densidade cellular $10^{6} \mathrm{cel} \cdot \mathrm{mL}^{-1}$ (a) e $10^{7} \mathrm{cel} \cdot \mathrm{mL}^{-1}$ (b) para as diferentes membranas (NF-90 e NF-270). 

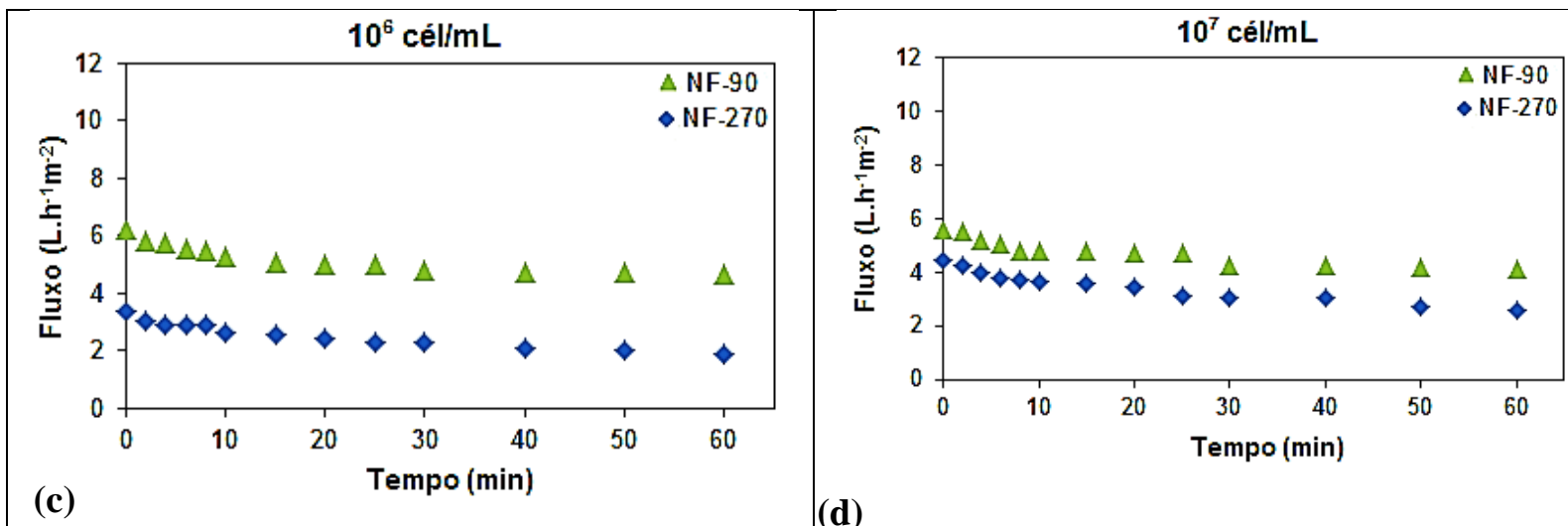

(d)

Figura 2 - Fluxo do permeado na pressão de 5 bar utilizando a Moringa $\mathrm{E}_{\mathrm{s}}$ com $\mathrm{KCl}(1 \mathrm{M})$ para as densidade cellular $10^{6} \mathrm{cel} \cdot \mathrm{mL}^{-1}$ (a) e $10^{7} \mathrm{cel} \cdot \mathrm{mL}^{-1}$ (b) para as diferentes membranas (NF-90 e NF-270).

Pode-se observar na Figura 1 e 2 que as amostra de água com baixa densidade celular $\left(10^{6} \mathrm{cel} \cdot \mathrm{mL}^{-1}\right)$, o fluxo médio do permeado para moringa com $\mathrm{NaCl}$ na membrana NF-90 foi de $3,4669 \mathrm{~L} / \mathrm{h}^{-1} \mathrm{~m}^{2}$ e para NF-270 foi de $2,1303 \mathrm{~L} / \mathrm{h}^{-1} \mathrm{~m}^{2}$. Com relação a moringa com $\mathrm{KCl}$ na membrana NF-90 foi de $5,2576 \mathrm{~L}^{-1} \mathrm{~m}^{2}$ e para NF-270 foi de $2,9250 \mathrm{~L} / \mathrm{h}^{-1} \mathrm{~m}^{2}$.

Para água com elevada densidade celular $\left(10^{7} \mathrm{cel} \cdot \mathrm{mL}^{-1}\right)$, o fluxo médio do permeado para moringa com NaCl na membrana NF-90 foi de $5,2575 \mathrm{~L}^{-1} \mathrm{~h}^{2}$ e para NF-270 foi de $4,4755 \mathrm{~L} / \mathrm{h}^{-1} \mathrm{~m}^{2}$. Com relação a moringa com $\mathrm{KCl}$ na membrana NF-90 foi de $4,7777 \mathrm{~L} / \mathrm{h}^{-1} \mathrm{~m}^{2}$ e para NF-270 foi de $3,4973 \mathrm{~L} / \mathrm{h}^{-1} \mathrm{~m}^{2}$.

De maneira geral, o fluxo do permeado da água tratada previamente com moringa usando $\mathrm{KCl}$ foi ligeralmente maior ao comparar com o $\mathrm{NaCl}$.

Com relação à redução deste fluxo durante o processo de filtração, pode-se atribuir à deposição de material sobre a superfície das membranas ou no interior dos poros, caracterizando fenômenos como a polarização por concentração e o fouling. Conforme descrito por Eagles e Wakeman (2002), pequenas partículas tendem a se depositar nas paredes do poro, ocasionando uma redução efetiva no diâmetro deste, reduzindo, dessa forma, o fluxo permeado. Quanto maior o diâmetro da partícula depositada na superfície da membrana, mais drástica tende a ser a redução do fluxo.

$\mathrm{Na}$ Tabela 4 apresentam as porcentagens do fouling das membranas N-90 e NF270 após o processo combinado $\mathrm{C} / \mathrm{F} / \mathrm{FAD}+\mathrm{NF}$ com solução salina de moringa com $\mathrm{NaCl}$ e $\mathrm{KCl}$.

Tabela 4 - Porcentagem de fouling da membrana NF-90 and NF-270

\begin{tabular}{ccccc}
\hline \multirow{2}{*}{$\begin{array}{c}\text { Densidadecelular } \\
(\text { cél } / \mathrm{mL})\end{array}$} & \multicolumn{2}{c}{ NF-90 fouling $(\%)$} & \multicolumn{2}{c}{ NF-270 fouling $(\%)$} \\
\cline { 2 - 5 } & $\begin{array}{c}\text { Moinga } \\
\text { com NaCl }\end{array}$ & $\begin{array}{c}\text { Moringa } \\
\text { com KCl }\end{array}$ & $\begin{array}{c}\text { Moringa } \\
\text { com NaCl }\end{array}$ & $\begin{array}{c}\text { Moringa } \\
\text { com KCl }\end{array}$ \\
\hline $10^{6}$ & 53,6 & 46.1 & 64,2 & 50.2 \\
$10^{7}$ & 42,2 & 35.2 & 53.6 & 48,9 \\
\hline
\end{tabular}

Comparando os dois coagulantes testados, a moringa com $\mathrm{NaCl}$ foi o que apresentou um maior entupimento, sendo que para água de baixa densidade celular, turbidez e cor, ofouling foi $>50 \%$.

$\mathrm{O}$ aumento da porcentagem de fouling em águas com turbidez menores pode ser explicado pelo fato destas apresentarem uma menor quantidade de sólidos totais suspensos, dificultando a formação de aglomerados no processo de 
coagulação/floculação, restando assim, maior quantidade de colóides responsáveis pelo entupimento dos poros da membrana.

Para comprovar a presença de incrustações na membrana, análises de microscopia eletrônica por varredura (MEV) da membrana anteriormente e posteriormente ao teste de nanofiltração foram realizadas e as fotografias da membrana antes e depois dos testes estão apresentadas na Figura 3.
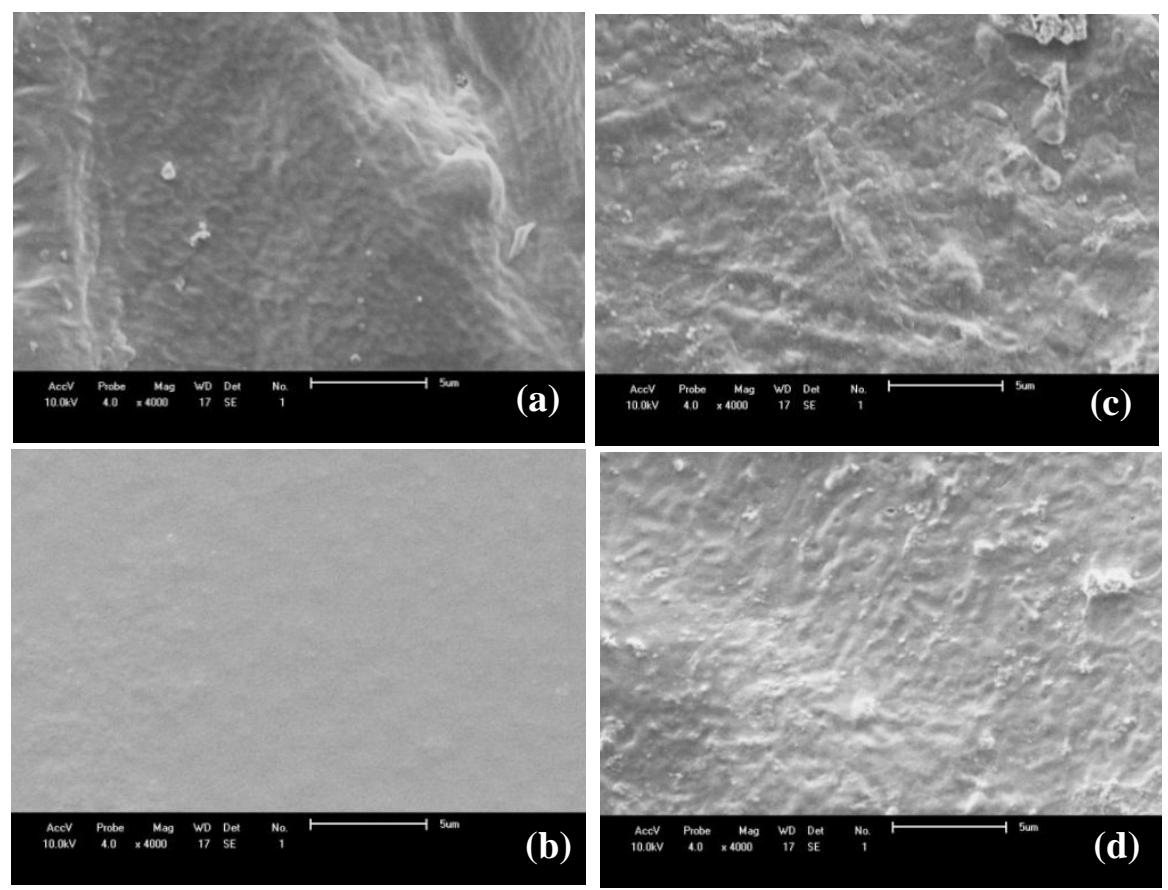

Figura 3 - Fotografias da superfície da membrana (a) NF- 90 and NF-270 (b), aumento 4000 vezes (a), (b) antes e (c), (d) depois do processo combinado C/F/FAD+NF.

Observa-se uma grande diferença entre as superfícies da membrana antes e depois dos testes de nanofiltração. Por meio da análise de MEV, uma espessa camada sobre a membrana, provavelmente de natureza orgânica, em função do coagulante utilizado, foi observada na superfície da membrana.

\section{CONCLUSÃo}

- A associação do processo de $\mathrm{C} / \mathrm{F} / \mathrm{FAD}$ com a nanofiltração através das membranas NF-90 e NF-270 apresentou valores de remoção de células de $M$. protocystis, turbidez e cor acima de $98 \%$, estando dentro dos padrões previstos pela legislação.

- A nanofiltração com a NF-90 e NF-270 demostrou-se eficiente na remoção de microcistina, após o tratamento combinado de $\mathrm{C} / \mathrm{F} / \mathrm{FAD}+\mathrm{NF}$, constatou-se que a concentração de microcistina no permeado está abaixo do valor permitido pela legislação de 1,0 $\mu \mathrm{g} / \mathrm{L}$ para os tempos de 0 e 30 minutos de filtração à pressão de 5 bar.

- A partir das conclusões expostas, pode-se contemplar que o processo combinado de $\mathrm{C} / \mathrm{F} / \mathrm{FAD}+\mathrm{NF}$ com Moringa oleifera em solução salina $\mathrm{NaCl}$ e $\mathrm{KCl}$ (1M) foi eficiente na remoção de cianobactérias e cianotoxinas. 


\section{REFERÊNCIA BIBLIOGRÁFICA}

ASSIS, Renata S. S. Remoção de Microcystis aeruginosa e microcistinas por flotação por ar dissolvido - estudo em escala de bancada utilizando sulfato de alumínio e cloreto férrico como coagulantes. 2006. 137 p. Dissertação (Mestrado em Tecnologia Ambiental e Recursos Hídricos) - Faculdade de Tecnologia, Universidade de Brasília, Brasília, 2006.

BAKER, R. W. Membrane Technology and Applications. $2^{\text {a }}$ ed. John Wiley \&Sons, Ltd. 2004. p. 4-7, 90-96.

BONGIOVANI, M. C., CAMACHO, F. P., NISHI, L., COLDEBELlA, P. F., VALVERDE, K. C., VIEIRA, A. M. S., BERGAMASCO, R., Improvement of the coagulation/flocculation process using a combination of Moringa oleifera Lam with anionic polymer in water treatment. Environmental Technology, 2014, http://dx.doi.org/10.1080/09593330.2014.899398

CARDOSO, K.C., BERGAMASCO,R., COSSICH, E.S., KONRADT-MORAES, L.C., Otimização dos tempos de mistura e decantação no processo de coagulação/floculação da água bruta por meio da Moringa oleifera Lam. Acta Scientiarum - Technology. 30, 193-198, 2008.

CHORUS, I., BARTRAM, J. Toxic Cyanobacteria in Water: A Guide to Their Public Health Consequences, Monitoring and Management, World Health Organization/E\&FN Spon/Routledge, London, 1999.

CORAL, L. A. Remoção de cianobactérias e cianotoxinas em águas d eabastecimento pela associação de flotação por ar dissolvido e nanofiltração. Florianópolis: UFSC. Dissertação Mestrado, 2009.

EAGLES, W.P.; WAKEMAN, R.J. 2002. Interactions between dissolved material and the fouling layer during microfiltration of a model beer solution. Journal of Membrane Science 206 (1-2), 253-264.

HITZFELD, Bettina C.; HOGER, Stefan J.; DIETRICH, Daniel R. Cyanobacterial toxin: removal during drinking water treatment, and human risk assessment. Environmental Health Perspectives, v. 108 (Suppl. I), p. 113-122, 2000.

JANSSENS, J.G., BUEKENS, A., Assessment of process selection for particle removal in surface water treatment. Journal Water SRT - Aqua; 42(5):279-88, 1993.

LÓPEZ-MUÑOZ, A. S., J. M. ARSUAGA, B. B. VAN DER, Influence of membrane solute and solution properties on the retention of phenolic compounds in aqueous solution by nanofiltration membranes. Separation and Purification Technology 66 (1) 194-652 201, 2009.

MADRONA, G.S.; SERPELLONI, G.B.; VIEIRA, A.M.S.; NISHI, L.; CARDOSO, K.C.; BERGAMASCO, R. Study of the Effect of saline Solution on the Extraction of the Moringa oleifera Seed's Active Component for Water Treatment. Water, Air and Soil Pollution 211 (1-4), 409-415, 2010.

NGHIEM, L. D. AND S. HAWKES, Effects of membrane fouling on the nanofiltration of 657 trace organic contaminants. Desalination 236 273-281, 2009.

RIBAU TEIXEIRA, M., M.J. ROSA, Comparing dissolved air flotation and conventional sedimentation to remove cyanobacterial cells of Microcystis aeroginosa. Part I: The key operating conditions. Separation and Purification Technology 52 (1) 8494, 2006a.

RIBAU TEIXEIRA, M., M. J. ROSA, Neurotoxic and hepatotoxic cyanotoxins removal by nanofiltration. Water Research 40 (15) 2837-2846, 2006 b.

SCHNEIDER, R. P.; TSUTIYA, M. T. Membranas filtrantes para o tratamento de água, esgoto e água de reuso. São Paulo: ABES, 234 p., 2001. 